\title{
Development and Evaluation of Multilead Wavelet-Based ECG Delineation Algorithms for Embedded Wireless Sensor Nodes
}

\author{
Francisco Rincón, Joaquin Recas, Nadia Khaled, Member, IEEE, and David Atienza, Member, IEEE
}

\begin{abstract}
This work is devoted to the evaluation of multilead digital wavelet transform (DWT)-based electrocardiogram (ECG) wave delineation algorithms, which were optimized and ported to a commercial wearable sensor platform. More specifically, we investigate the use of root-mean squared (RMS)-based multilead followed by a single-lead online delineation algorithm, which is based on a state-of-the-art offline single-lead delineator. The algorithmic transformations and software optimizations necessary to enable embedded ECG delineation notwithstanding the limited processing and storage resources of the target platform are described, and the performance of the resulting implementations are analyzed in terms of delineation accuracy, execution time, and memory usage. Interestingly, RMS-based multilead delineation is shown to perform equivalently to the best single-lead delineation for the 2-lead QT database (QTDB), within a fraction of a sample duration of the Common Standards for Electrocardiography (CSE) committee tolerances. Finally, a comprehensive evaluation of the energy consumption entailed by the considered algorithms is proposed, which allows very relevant insights into the dominant energy-draining functionalities and which suggests suitable design guidelines for long-lasting wearable ECG monitoring systems.
\end{abstract}

Index Terms-Ambulatory electrocardiogram, delineation, digital wavelet transform, energy-constrained systems, multilead, wireless sensor node.

\section{INTRODUCTION}

A CCORDING to the World Health Organization, cardiovascular diseases are the major cause of mortality worldwide, responsible for one third of deaths worldwide, in 2004, and economic fallout in billions of dollars [1]. Their burden is only expected to rise due to, on the one hand, the fast aging of the world population, and on the other hand, the increasing prevalence of unhealthy lifestyles. Cardiovascular diseases

Manuscript received February 13, 2011; revised June 20, 2011; accepted July 26,2011 . Date of publication August 8, 2011; date of current version November 23,2011 . This work was supported by the Spanish Government Research Grant TIN2008-00508.

F. Rincón is with the School of Engineering, Ecole Polytechnique Fédérale de Lausanne (EPFL), 1015 Lausanne, Switzerland, and also with the Department of Computer Architecture and Automation, Complutense University of Madrid, 28040 Madrid, Spain (e-mail: francisco.rincon@fdi.ucm.es).

J. Recas is with the Department of Computer Architecture and Automation, Complutense University of Madrid, 28040 Madrid, Spain (e-mail: jrecas@fis.ucm.es).

N. Khaled and D. Atienza are with the School of Engineering, Ecole Polytechnique Fédérale de Lausanne (EPFL), 1015 Lausanne, Switzerland (e-mail: nadia.khaled@epfl.ch; david.atienza@epfl.ch).

Color versions of one or more of the figures in this paper are available online at http://ieeexplore.ieee.org.

Digital Object Identifier 10.1109/TITB.2011.2163943 require close and potentially continuous medical supervision and care. They are thus expected to soon require healthcare costs and medical management needs that are unsustainable for traditional healthcare delivery systems. Wireless body sensor network (WBSN) technologies are poised to offer large-scale and cost-effective solutions to this problem. The use of wearable, miniaturized, and wireless sensors, able to continuously measure and wirelessly report cardiac signals, can indeed provide the ubiquitous, long-term and even real-time monitoring required by the patients, as well as its integration with the patient's medical record and its coordination with nursing/medical support.

Among the relevant cardiac signals, the noninvasive electrocardiogram (ECG) has long been used as a means to diagnose diseases reflected by disturbances of the heart's electrical activity. Beyond traditional electrocardiography, the automated processing and analysis of the ECG signal has been receiving significant attention and has witnessed substantial advances [2], [3]. In particular, a large body of algorithms have been proposed for the detection of the ECG characteristic waves, so-called ECG delineation, following a variety of approaches based on lowpass differentiation [4], the wavelet transform (WT) [5]-[7], dynamic time warping [8], artificial neural networks [9], hidden Markov models [10], or morphological transforms [11]. The morphological and timing information of the detected waves, namely the QRS complex and $\mathrm{P}$ and $\mathrm{T}$ waves, can be used to diagnose many cardiac ailments.

Traditionally, the automatic analysis of ECG signals, including delineation, was either taking place online on bulky, highperformance bedside cardiac monitors, or performed offline during a postprocessing stage after ambulatory ECG recording using wearable, yet obtrusive, ECG data loggers (Holter devices). Recently, however, a significant industrial and academic effort has been dedicated to online automatic ECG analysis on miniature, wearable and wireless ECG monitors as an enabler of nextgeneration mobile cardiology systems. These efforts essentially resulted in the development of two commercial products and a research prototype: Toumaz's Sensium Life Pebble [12], a CEcertified ultra-small and ultra-low-power monitor for single-lead ECG, heart rate (HR), physical activity, and skin temperature measurements with a reported autonomy of five days on a hearing aid battery; Corventis's PiiX [13], a CE and FDA-cleared lead-less band-aid-like ECG sensor able to perform continuous arrhythmia detection based on HR measurements; and finally IMEC's prototype of a single-lead bipolar ECG patch [14] for ambulatory HR monitoring with a claimed 10-day autonomy 
on a $160 \mathrm{mAh}$ Li-ion battery. Accordingly, state-of-the-art unobtrusive wireless mobile/ambulatory ECG monitors are single lead and limited to embedded HR measurement and analysis. Therefore, this work comprehensively assesses the feasibility of a full online automated multilead ECG delineation on a stateof-the-art small wireless wearable sensor platform, i.e., Shimmer [15].

More specifically, this work revisits the originally offline WTbased ECG delineation algorithm, first introduced in [5] and further developed and extensively evaluated in [7], and which we previously nonstraightforwardly optimized and ported for real-time single-lead ECG delineation [16] on the Shimmer embedded sensor node. It then extends our online WT-based single-lead delineation algorithm [16] for the multilead scenario, including the prerequisite baseline removal filtering and the sensible algorithmic modifications and parameter optimization to achieve real-time execution on the Shimmer notwithstanding its limited processing and storage resources. As a matter of fact, an embedded wireless sensor node typically has only a few kilobytes of memory [12], [14], [15] (e.g., $10 \mathrm{kB}$ for Shimmer), is equipped with an ultra-low-power microcontroller running at a maximum clock speed between 8 and $16 \mathrm{MHz}$, and does not include hardware support for division and floating-point operation. Due to these stringent hardware limitations, the development and optimization of the herein presented delineation algorithms for their real-time execution on a WBSN platform entails two main challenges: 1) maintaining good delineation results while performing the delineation in real time on mobile cardiology devices; and 2) minimizing the system's energy consumption through reducing the CPU execution time and the necessary wireless data communication.

The rest of the paper is organized as follows. Section II presents the delineation algorithms investigated in this work, while Section III details the performance metrics and the ECG database subsequently used for their performance comparison. Then, in Section IV, the implementation of these algorithms on the Shimmer node is described, and their performance evaluation is provided. A comparative study of the energy consumption (and corresponding sensor node lifetime) entailed by the proposed algorithms for a realistic wearable ECG monitoring scenario is further performed in Section V. Finally, the conclusions of this work are summarized in Section VI.

\section{WT-BASED ECG DELINEATION METHODS}

\section{A. State-of-the-Art Single-Lead Offline Delineation Algorithm}

As aforementioned, this work is based on the offline WTbased ECG delineator described and validated in [7]. As such, the therein detailed description and discrete-time implementation of the underlying dyadic wavelet transform is not herein reproduced. For self-containment, it is sufficient to mention that this delineator performs the detection of all characteristic points (onset, peak, and end) of the ECG waves using a quadratic spline WT, which produces derivatives of smoothed versions of the input ECG signal at five dyadic scales (i.e., $2^{1}$ to $2^{5}$ ). The choice of these scales was motivated by the observation that most of the energy of the ECG signals lies within these scales [7]. In

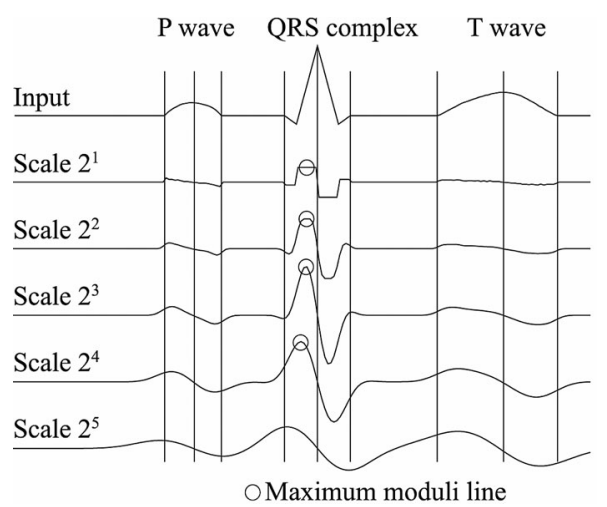

Fig. 1. WT at the first five scale of an ECG-like signal.

particular, it was shown that the energy of the QRS complex is lower in scales higher than $2^{4}$, and that the $\mathrm{P}$ and $\mathrm{T}$ waves have significant components at scale $2^{5}$. These observations are illustrated in Fig. 1, which depicts an ECG-like signal and the five first scales of its WT.

Fig. 1 can also be used to illustrate the WT-based ECG delineation principle. The WT at scale $2^{k}$ is proportional to the derivative of the filtered version of the input ECG signal with a smoothing function at scale $2^{k}$. Then, the zero crossings of the WT correspond to the maxima or minima of the smoothed ECG signal at different scales, and the maximum absolute values of the WT are associated with maximum slopes in the smoothed ECG signal. This is verified in Fig. 1, where monophasic waves ( $\mathrm{P}, \mathrm{R}$, and $\mathrm{T}$ waves) produce a positive maximum-negative minimum pair across scales, with a zero crossing in between. Moreover, each sharp change in the input ECG signal is associated with a line of maxima or minima across the scales. Accordingly, using this information of local maxima, minima, and zero crossings at different scales, the WT-based algorithm identifies the fiducial points of the ECG signal. The detailed description of this algorithm is deferred to Section IV-B.

\section{B. Multilead Delineation}

In clinical practice, however, several leads are simultaneously acquired, be it the standard 12-lead ECG in traditional clinical settings or the 3-lead configuration in emerging ambulatory ECG monitoring. This calls for the deployment of delineation approaches able to exploit the multiple available leads to improve the accuracy, stability, and resilience to artifacts of the characteristic waves measurements, compared to single-lead delineation.

There are two approaches to multilead delineation. The first approach consists in first delineating each and every lead individually, then applying a median postprocessing selection rule over the characteristic wave peaks, to select the "best" delineated ones. Then, the wave boundaries with longest wave duration are chosen, with some outlier protection, in order to retain the "best" global single-lead delineation [4], [7]. When only two leads are available, this approach is, however, not applicable, and must be replaced by a "genie" selection that chooses for each point the lead with less error. However, this "genie" selection, which 
represents the optimal delineation, can only be used for validation purposes since the algorithm cannot know which is the lead with less error. The second approach instead proposes to combine the individual leads into a multilead signal-which provides an overall view of the cardiac phenomena and is independent of the lead system used-on which a single-lead delineation algorithm is further applied. Different multilead signals can be found in the literature: 1) the combination of the ECG leads in the root mean squared (RMS) sense [17]-[19]; 2) a derived spatial lead obtained via a multistep iterative search on the spatial WT loops of the two or three original orthogonal leads [20]; 3) a combination of the derivatives of the ECG leads [21]. Given the limited processing and storage resources of our target embedded sensor node, we herein retain the wellestablished RMS-based multilead delineation approach due to its lower computational complexity and better scalability. We will additionally benchmark its performance with respect to the selection-based multilead delineation.

Accordingly, the available $L$ ECG leads $x_{l}[n]$, with $l=$ $1, \ldots, L$, are first combined in a single multilead signal $x_{\mathrm{RMS}}[n]$ :

$$
x_{\mathrm{RMS}}[n]=\sqrt{\frac{1}{L} \sum_{l=1}^{L} x_{l}^{2}[n]}
$$

where $n$ denotes the discrete-time index, on which single-lead delineation is performed. For a meaningful combination, it is crucial to remove baseline wander (mainly caused by respiration, electrode impedance changes due to perspiration and body movements) on each of the leads before computing the RMS [17], [18]. Therefore, since the quality of the subsequent delineation depends on the baseline wander correction, we assess in this work the effectiveness of the following two state-ofthe-art approaches.

1) Cubic Spline Baseline Estimation [22]: This method uses a third-order polynomial to approximate the baseline wander, which is then subtracted from the original signal. To do so, a representative sample (or knot) is chosen for each beat from the silent isoelectric line, which is represented by the PQ segment in most heart rhythms. The polynomial is then fitted by requiring it to pass through successive triplets of knots.

2) Morphological Filtering [23]: This method applies several erosion and dilation operations to the original ECG signal to estimate the baseline wander. It first applies an erosion followed by a dilation, which removes peaks in the signal. Then, the resultant waveforms with pits are removed by a dilation followed by an erosion. The final result is an estimate of the baseline drift. The correction of the baseline is then done by subtracting this estimate from the original signal.

\section{Database And PERformance Metrics}

We validate the considered delineation algorithms using the 105 records of the QT database (QTDB) [24]. The QTDB is a free-access database, which consists of 15-minute excerpts of two-lead ECG recordings sampled at $250 \mathrm{~Hz}$, with manual annotations of the QRS complexes, $\mathrm{P}$ and $\mathrm{T}$ waves performed by expert cardiologists. These annotations span a total of 3600 beats, and feature a wide variety of ECG morphologies.

To assess the performance of the investigated algorithms, the following rules are applied. An automatic annotation is considered to be related to a manual one if their time interval is smaller than $320 \mathrm{~ms}$. This value has been empirically chosen to compare our results to the ones of [7] as fairly as possible, since all the proposed algorithms are based on this work. This value allows us to obtain the closest results of sensitivity and positive predictivity to [7] and, therefore, be able to directly compare the mean error and standard deviation values. Hence, this pair of annotations is a true positive. Then, for the "genie" selection scheme, when two automatic annotations on different leads fall within that interval, the closest one to the manual annotation is chosen to compute the error, and the other one is not counted as a false positive. The reported error statistics correspond to the computation of (automatic-manual) marks. Finally, each manual annotation that has no corresponding automatic annotation is counted as a false negative, and automatic annotations without manual annotations count as false positive. Accordingly, the sensitivity $(S e)$ and positive predictivity $(P+)$ of a delineation algorithm are defined as [25]:

$$
S e=\frac{T P}{T P+F N}, \quad P+=\frac{T P}{T P+F P}
$$

where $T P$ is the number of true positive detections, $F N$ is the number of false negative detections and, $F P$ is the number of false positive detections. In addition, due to the QTDB format, it is impossible to know if the lack of an annotation means that the wave is not present or that the cardiologist did not annotate the point with confidence for some reason. In the first case, an automatic detection would count as a false positive, but in the second case, it would not necessarily mean that the detection is erroneous. As a result of this overestimation of false positives, we show in the tables the value of $P_{\min }^{+}$, which is a lower bound on the real value of $P^{+}$.

Moreover, the mean $(m)$ and the standard deviation of the delineation error $(\sigma)$ are also computed. The mean error $(m)$, which is computed as the average of the errors across all the records, shows how close the results of the algorithm are to those that have been manually annotated in the database. Finally, the standard deviation of the error $(\sigma)$, which is defined as the average of the standard deviation of each record, provides us information about the stability of the detections.

\section{REAL-TIME EMBEDDED DELINEATION}

This section first describes the target-embedded sensor platform, on which our proposed delineation algorithms will be implemented and validated. Since the original delineation algorithms of Section II cannot be executed in real-time on this platform, we subsequently detail the algorithmic adaptations and the platform-dependent optimizations that we carried out to achieve real-time execution as well as optimized processing and memory footprint. 


\section{A. The Shimmer Embedded Wearable Sensor Platform}

Our target WBSN node is the Shimmer platform [15]. This platform is equipped with the TI MSP430F1611 [26], an ultralow-power 16-bit microcontroller, which offers a maximum frequency of $8 \mathrm{MHz}$, includes $10 \mathrm{kB}$ of RAM, $48 \mathrm{kB}$ of Flash, and some peripherals such as an 8-channel analog-to-digital (A/D) converter, as well as a direct memory access unit (DMA) and a fast hardware multiplier. This platform also provides two radio chips (Bluetooth and IEEE 802.15.4-compliant), a 3-axis accelerometer and an expansion port used to connect a daughter board equipped with additional sensors (ECG, gyroscopes, etc.). The daughter board used in this work is able to acquire and condition 3-lead ECGs.

This sensor platform has been designed to be programmed with the TinyOS [27] operating system and its specific drivers. Unfortunately, TinyOS is not suitable for the applications presented in this paper, since it does not feature hard real-time capabilities, which are required for ECG sampling, the execution of the delineation algorithms and, especially, the wireless communication. Due to these limitations, we ported FreeRTOS [28], which is a portable, open source, hard real-time mini kernel that includes support for the TI MSP430 microcontroller and the CC2420 IEEE 802.15.4-compliant radio chip used by Shimmer. In addition, this operating system can be customized to reduce the program memory usage. This possibility leads to the creation of very light prioritized multitask real-time applications in which the scheduler puts the microcontroller in low-power mode when none of these tasks is being executed. Finally, we have used Code Composer Essentials (CCE) v.3.1 [29], which uses all the hardware resources of the target microcontroller, to compile the proposed algorithms for the node.

\section{B. Optimized Online Single-Lead Implementation}

As aforementioned in Section II-A, the baseline single-lead offline delineation algorithm [5], [7] performs a multiscale analysis on the computed first five scales of the DWT of the input ECG signal, in order to extract the corresponding ECG fiducial points. More specifically, the main wave of the QRS complex is detected. Then, this complex is delineated, which consists in detecting its secondary peaks and finding its onset and end. Finally, the $\mathrm{P}$ and $\mathrm{T}$ waves are detected along with their respective onset and end.

To make this baseline algorithm online, as well as to improve its delineation performance, we previously introduced several modifications and new decision rules [16]. In particular, we adopted the ECG detection thresholds, sampling sizes, and search windows to enable the real-time implementation of the proposed algorithm on a state-of-the-art commercial embedded sensor platform. Since these modifications and novel decision rules are relevant for the herein proposed multilead extension, they are summarized in this section. Note that, for all the following, the ECG signal is sampled at a frequency of $250 \mathrm{~Hz}$ (i.e., sampling period of $4 \mathrm{~ms}$ ), which corresponds to the sampling frequency of the used QT database.

1) QRS Detection: Like [5], [7], we detect the QRS main wave by looking for a pair of absolute maximum moduli lines of opposite signs present across scales $2^{4}$ to $2^{1}$, and respectively exceeding the predefined thresholds $\epsilon_{Q R S}^{4}$ to $\epsilon_{Q R S}^{1}$. This corresponds to searching for a maximum modulus at scale $2^{4}$, then for one in its neighborhood at scale $2^{3}$, and so on for lower scales. Note that a maximum modulus at scale $2^{k}$ is considered to be in the neighborhood of one at scale $2^{k-1}$, if the interval between them is less than 10 samples. In case more than one maximum modulus lie within this interval, we simply select the closest one. Furthermore, the rules in [5] are applied to eliminate redundant lines and to choose the most significant pair of maximum moduli lines. Both positive and negative $\mathrm{R}$ waves are allowed, and the peak of the main wave of the QRS complex is found at the zero crossing between the two maximum moduli at scale $2^{1}$. Our optimized rules for computing the amplitude thresholds $\epsilon_{Q R S}^{k}$, the estimate of the R-R interval, and the search-back procedure when no QRS complex is detected, can be found in [16].

2) QRS Delineation: Once the main wave of the QRS complex is detected, we similarly delineate the secondary waves around it. Accordingly, a secondary wave is also characterized by a pair of maximum moduli with opposite signs across scales, and the wave peak is found at the zero crossing at scale $2^{1}$ between these two maximum moduli. More specifically, we search for maximum moduli at scale $2^{2}$ before and after the main wave, in a window starting 20 samples (i.e., $80 \mathrm{~ms}$ ) before the main wave and ending 40 samples (i.e., $160 \mathrm{~ms}$ ) after it, respectively exceeding the optimized thresholds $\gamma_{Q R S_{\text {pre }}}$ and $\gamma_{Q R S_{\text {post }}}$ [16]. As in [7], we only take three waves into account. Thus, if more than three significant waves are detected (i.e., the main wave plus two secondary waves), we select the closest ones relatively to the main peak.

The onset (resp. end) of the QRS complex is before (resp. after) the first (resp. last) significant slope of the QRS, which is associated with the above-identified maximum of moduli at scale $2^{2}$. Let $n_{\text {first }}$ (resp. $n_{\text {last }}$ ) be the position of the first (resp. last) maximum modulus at scale $2^{2}$ associated with any wave of the QRS complex. Then, looking at scale $2^{4}$, the onset (resp. end) of the QRS complex is the first sample before $n_{\text {first }}$ (resp. after $\left.n_{\text {last }}\right)$ whose value is below $\xi_{Q R S_{\text {onset }}}\left(\right.$ resp. $\xi_{Q R S_{\text {end }}}$ ). The detailed expressions of $\xi_{Q R S_{\text {onset }}}, \xi_{Q R S_{\text {end }}}$, and the search windows for $n_{\text {first }}$ and $n_{\text {last }}$ are given in [16].

3) $P$ and T Waves Delineation: $\mathrm{P}$ and $\mathrm{T}$ waves' detection and delineation follow similar procedures as those previously described for the QRS complex. As suggested in [7], we first look for maximum moduli at scale $2^{4}$ within a search window. [7] also defines thresholds $\epsilon_{P / T}^{4}$ above which maximum moduli are considered significant. Since it turned out that these thresholds were often close to 0 , and that considering every positive maximum and negative minimum gives better results, we simply detected all maximum moduli. Then, we further consider those with amplitude greater than a threshold $\gamma_{P / T}$ [16] within a search window that is defined for each wave specifically. If no suitable pair is found at scale $2^{4}$, we repeat the above procedure at scale $2^{5}$. Once the pair of maximum moduli is found at scale $2^{k}(k=4$ or 5$)$, we select the zero crossing between them at scale $2^{3}$ as the wave peak. If no such zero crossing is found, we 
TABLE I

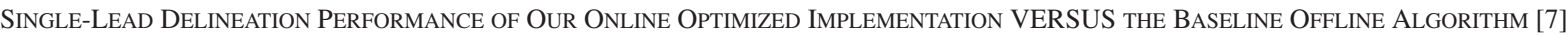

\begin{tabular}{|c|c|c|c|c|c|c|c|c|}
\hline Method & Parameter & $P_{\text {onset }}$ & $P_{\text {peak }}$ & $P_{\text {end }}$ & $Q R S_{\text {onset }}$ & $Q R S_{\text {end }}$ & $T_{\text {peak }}$ & $T_{\text {end }}$ \\
\hline this work & Se $(\%)$ & 99.87 & 99.87 & 99.91 & 99.97 & 99.97 & 99.97 & 99.97 \\
\hline 16-bit int & $P_{\min }^{+}(\%)$ & 91.98 & 92.46 & 91.70 & 98.61 & 98.72 & 98.91 & 98.50 \\
\hline Shimmer ${ }^{\mathrm{TM}}$ & $m \pm \sigma(\mathrm{ms})$ & $8.6 \pm 11.2$ & $10.1 \pm 8.9$ & $0.9 \pm 10.1$ & $3.4 \pm 7.0$ & $3.5 \pm 8.3$ & $3.7 \pm 13.0$ & $-2.4 \pm 16.9$ \\
\hline Martinez [7] & $\begin{array}{c}\text { Se }(\%) \\
P_{\min }^{+}(\%) \\
m \pm \sigma(\mathrm{ms})\end{array}$ & $\begin{array}{c}98.87 \\
91.03 \\
2.0 \pm 14.8\end{array}$ & $\begin{array}{c}98.87 \\
91.03 \\
3.6 \pm 13.2\end{array}$ & $\begin{array}{c}98.75 \\
91.03 \\
1.9 \pm 12.8\end{array}$ & $\begin{array}{c}99.97 \\
\text { N/A } \\
4.6 \pm 7.7 \\
\end{array}$ & $\begin{array}{c}99.97 \\
\text { N/A } \\
0.8 \pm 8.7\end{array}$ & $\begin{array}{c}99.77 \\
97.79 \\
0.2 \pm 13.9\end{array}$ & $\begin{array}{c}99.77 \\
97.79 \\
-1.6 \pm 18.1\end{array}$ \\
\hline \multicolumn{2}{|c|}{ Tolerances $\left(2 s_{C S E}\right)$} & 10.2 & - & 12.7 & 6.5 & 11.6 & - & 30.6 \\
\hline
\end{tabular}

The baseline offline algorithm [7].

use instead the zero crossing at scale $2^{k}$. The onset and end are then detected using the same principles as for the QRS onset and end. Our optimized delineation procedures for the $\mathrm{P}$ and $\mathrm{T}$ waves are detailed in [16] and are not reproduced here for conciseness.

4) Code Optimizations for Real-Time Execution: Since the MSP430 microcontroller does not have a floating-point unit, the first adaptation we performed to port and run in real-time our ECG delineation algorithm in Shimmer was the conversion of all the operations from the reference implementation on the PC to use 16-bit signed integers instead of floating-point numbers. Then, since the amount of available memory in the node is limited to $10 \mathrm{kB}$, the second code adaptation consisted in restricting the maximum buffer size for the input data and each of the five wavelet transforms to 512 samples. Since each sample uses a 16-bit integer (two bytes), the total amount of data used is $6 \mathrm{kB}$ of RAM. Overall, only $6.5 \mathrm{kB}$ of RAM are used for temporary data in our implementation of the online single-lead delineation algorithm, and the instructions require about $15 \mathrm{kB}$ of Flash memory. Thus, making the algorithm suitable for current lowpower sensor node platforms.

Then, the third transformation we applied into the reference implementation was related to the computation of the amplitude thresholds $\epsilon$. These thresholds are originally computed using the RMS of the data blocks, which requires a square root operation. However, the $\mathrm{C}$ library sqrt function used by the CCE compiler to implement the RMS requires many floating-point operations. Thus, we replaced this function with our own implementation of the Longhand square root algorithm of [30], which performs well on platforms without hardware division support. Overall, we fully avoided all time-consuming software implementations of floating-point operations at the compiler level for our algorithm.

The fourth and final optimization step for real-time embedded implementation was the optimization of the generated code by the CCE compiler for the MSP430. In particular, the most timeconsuming parts of our proposed delineation algorithm are the low-pass and high-pass filters required for the computation of the five scales of the DWT [7], which are called four and five times, respectively, for each input data sample. However, the multiplications and divisions entailed by these filters can be replaced by fast shift and addition operations. Therefore, we generated few lines of assembly code to replace the generated code by the CCE compiler from the original $\mathrm{C}$ code of our algorithm.
5) Validation and Experimental Results: For this first set of experiments, we run our improved online single-lead delineation algorithm on the Shimmer platform and compute the four relevant performance metrics described in Section III. Table I reports the obtained results, together with those of the original offline single-lead algorithm [7]. The third row indicates the standard deviation tolerances by the CSE working party [31]. Note that for the sack of consistency with the validation procedure of [7], the results of our online algorithm in Table I correspond to performing online single-lead delineation on the two channels of each QTDB excerpt, then selecting the automatic annotation on the channel producing less error, for each manual annotation of the database. The results obtained using this validation procedure represent the optimal single-lead delineation that can be achieved using a two-channel ECG input signal, which we previously labeled "genie" selection.

Table I illustrates that our implementation performs within the standard deviation tolerances for all points, except the $\mathrm{QRS}_{\text {onset }}$ and $\mathrm{P}_{\text {onset }}$, for which we are above the agreed tolerances by only a fraction of the sample duration. Furthermore, Table I shows that this implementation of our proposed algorithm consistently outperforms the state-of-the-art offline algorithm proposed in [7] in terms of standard deviation, while running in real time and preserving a high sensitivity and predictivity.

In addition, we measured the real-time performance of our algorithm implementation on the Shimmer platform. To this end, we used an internal timer of the MSP430 to compute the total processing time. This timer is suspended when data transfers are performed through the serial port, and resumed when the data are being processed, as if it were in real-time. Thus, we can extract a ratio between the amount of data to process and the actual time needed to process the data of the ECG waves of the QDTB. Interestingly, the obtained processing ratio results using the proposed algorithm and embedded implementation prove that we can perform real-time delineation while keeping the MSP430 microcontroller in sleep mode about 93\% of the time on average. Thus, enabling a very low-power embedded ECG wave delineation system.

\section{Optimized Online Multilead Delineation}

As aforementioned, the considered multilead delineation algorithms are all based on the above-validated online WT-based single-lead delineator. Therefore, no further optimizations are needed in the delineation stage. The baseline wander filtering of each lead prior to the RMS combination must be however 
TABLE II

PERFormance COMPARISON OF ONLINE MULTILEAD AND Single-LEAD DELINEATION

\begin{tabular}{|c|c|c|c|c|c|c|c|c|}
\hline Method & Parameter & $\overline{P \text { onset }}$ & $\bar{P} P_{\text {peak }}$ & $P_{\text {end }}$ & $\overline{Q R S_{\text {onset }}}$ & $Q R S_{\text {end }}$ & $T_{\text {peak }}$ & $T_{\text {end }}$ \\
\hline 2-lead & $\mathrm{Se}(\%)$ & 94.02 & 94.02 & 94.05 & 99.67 & 99.67 & 98.00 & 97.94 \\
\hline 16-bit int & $P_{\min }^{+}(\%)$ & 95.15 & 95.42 & 95.49 & 99.23 & 99.20 & 99.23 & 98.72 \\
\hline Cubic spline filter & $m \pm s(\mathrm{~ms})$ & $4.1 \pm 17.1$ & $12.8 \pm 12.1$ & $-2.1 \pm 14.2$ & $3.2 \pm 8.7$ & $7.3 \pm 11.9$ & $1.9 \pm 17.4$ & $-4.1 \pm 25.3$ \\
\hline 2-lead & $\mathrm{Se}(\%)$ & 96.24 & 96.24 & 96.27 & 99.75 & 99.75 & 98.33 & 98.11 \\
\hline 16-bit int & $P_{m i n}^{+}(\%)$ & 91.19 & 91.38 & 91.55 & 97.07 & 97.07 & 98.58 & 98.08 \\
\hline Morphological filter & $m \pm s(\mathrm{~ms})$ & $2.5 \pm 16.4$ & $14.7 \pm 13.4$ & $-1.9 \pm 15.5$ & $6.9 \pm 8.0$ & $9.0 \pm 9.1$ & $5.3 \pm 19.3$ & $-11.4 \pm 24.9$ \\
\hline Single-lead & $\mathrm{Se}(\%)$ & 98.84 & 98.84 & 98.87 & 99.61 & 99.61 & 99.35 & 99.32 \\
\hline 16-bit int & $P_{\min }^{+}(\%)$ & 92.66 & 92.93 & 93.24 & 99.56 & 99.56 & 99.49 & 99.24 \\
\hline Lead 1 & $m \pm s(\mathrm{~ms})$ & $13.4 \pm 14.8$ & $15.6 \pm 12.1$ & $1.6 \pm 13.2$ & $5.4 \pm 8.4$ & $1.5 \pm 10.1$ & $5.3 \pm 18.4$ & $-5.3 \pm 22.7$ \\
\hline Single-lead & Se $(\%)$ & 97.31 & 97.09 & 96.81 & 99.67 & 99.72 & 99.35 & 99.18 \\
\hline 16-bit int & $P_{\min }^{+}(\%)$ & 91.76 & 92.21 & 91.43 & 98.61 & 98.72 & 98.90 & 98.38 \\
\hline Lead 2 & $m \pm s(\mathrm{~ms})$ & $10.4 \pm 19.4$ & $6.9 \pm 16.9$ & $-8.5 \pm 17.9$ & $8.6 \pm 12.6$ & $8.7 \pm 13.1$ & $3.5 \pm 23.5$ & $-4.6 \pm 27.2$ \\
\hline \multicolumn{2}{|c|}{ Tolerances $\left(2 s_{C S E}\right)$} & 10.2 & - & 12.7 & 6.5 & 11.6 & 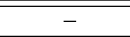 & 30.6 \\
\hline
\end{tabular}

optimized in view of its implementation on our target platform, as subsequently described.

1) Cubic Spline Baseline Estimation: For an improved estimation, we select as "knot" a point within the PR segment. More specifically, we experimentally choose the point that is 28 ms (seven samples) before the beginning of the QRS complex. Consequently, detecting a "knot" boils down to detecting the beginning of the QRS complex, using a simplified version of the single-lead delineator. Then, once three knots are detected, we use these points to fit a third-order polynomial, which provides an approximation of the baseline wander. This approximation is further subtracted from the original signal.

As previously shown, the performance of single-lead delineation is robust to the reduction in precision induced by the conversion of all operations to use 16-bit integers instead of floating-point operands. On the contrary, it turned out that the calculation of the coefficients of the third-order polynomial needed to approximate the baseline wander is highly affected by this conversion. Therefore, we had to use 32-bit operands to calculate and store the coefficients, which doubles the execution time of the filtering stage.

2) Morphological Filtering: It is based on the sequential application, on the input signal, of the dilation $(\oplus)$ and erosion $(\ominus)$ operators defined as [23]:

$(f \oplus B)(n)=\max _{m=0, \ldots, M-1}\left\{f\left(n-\frac{M-1}{2}+m\right)+B(m)\right\}$

$(f \ominus B)(n)=\min _{m=0, \ldots, M-1}\left\{f\left(n-\frac{M-1}{2}+m\right)-B(m)\right\}$

$$
\text { for } n=\left\{\frac{M-1}{2}, \ldots, N-\frac{M+1}{2}\right\}
$$

where $f(n),\{n=0,1, \ldots, N-1\}$ denotes a discrete-time signal consisting of $N$ points, and $B(m),\{m=0,1, \ldots, M-1\}$ is a symmetric structuring element of $M$ points. Essentially, the two morphological operators consist of a vector addition/subtraction, followed by a search of a maximum or a minimum over the resultant vector. The length of the structuring elements, which is set according to the duration of the shortest ECG characteristic wave to be detected and the sampling frequency, is either 50 or 75 , depending on the operation to be applied. The large size of the structuring elements results into long linear searches to find the maxima and minima of (3) and (4), which are quite consuming in terms of computation. However, it turns out that the structuring elements related to baseline wander removal are horizontal line segments with zero amplitude. Equations (3) and (4) thus correspond to linear searches to find the maximum and the minimum of the input signal $f(n)$ over a symmetric window of length $M$ around the discrete-time instant $n$. From one discrete-time instant to the next, $M-1$ elements of this window will then remain the same. Consequently, it is sufficient to store these elements in intermediate buffers and use two indexes to keep track of the maximum and minimum of the considered segment. This optimization reduces the number of linear searches by $92 \%$, therefore significantly speeding up the calculations.

3) Validation and Experimental Results: The accuracy of our online multilead delineation using the two considered baseline wander removal techniques, running on our target platform for the QTDB database, are compiled in Table II. For comparison, this table also includes the delineation results of the online single-lead delineation algorithm applied on each of the two leads of the database.

Comparing the accuracy of single-lead delineation on individual leads (last two rows of Table II) and that of the multilead approach based on the "best"-lead selection after single-lead delineation (first row of Table I), it is confirmed that exploiting the multiple leads has the potential to provide more accurate boundary locations than any lead by itself. Considering the RMS-based multilead approach, the two considered baseline removal techniques exhibit equivalent accuracy figures in terms of mean $m$ and standard deviation $s$, which are off the tolerances by only a fraction of the sample duration for all wave locations, except the onset of the $\mathrm{P}$ wave. For this particular wave boundary, none of the methods discussed in this paper fulfills the loose criterion on the tolerance for the dispersion of automatic delineation errors. Interestingly, multilead delineation with cubic spline baseline estimation offers the best positive predictivity performance, $P_{\min }^{+}$, among all the methods of Table II.

Furthermore, Table II also shows that multilead delineation performs equivalently to the best single-lead delineation (i.e., obtained on lead 1), and outperforms the worst single-lead delineation (i.e., obtained on lead 2) by one sample duration. This 
TABLE III

COMPUTATIONAL BURDEN AND MEMORY FOOTPRINT

\begin{tabular}{c|c|c}
\hline \hline Algorithm & RAM usage $(\mathrm{kB})$ & Execution time $(\%)$ \\
\hline Single lead & 7.2 & 6.78 \\
\hline $\begin{array}{c}\text { 2-lead RMS } \\
\text { w/ cubic spline filtering }\end{array}$ & 14.9 & 33.22 \\
\hline $\begin{array}{c}\text { 2-lead RMS } \\
\text { worph. filtering }\end{array}$ & 10.9 & 19.17 \\
\hline
\end{tabular}

further confirms the previously introduced evidence [18] that RMS-based lead combining alleviates the dependency of delineation results on specific leads or lead systems, and that it provides a more robust and stable boundary locations than single-lead delineation. The validation herein produced is limited to 2-lead ECG excerpts of the QTDB database, due to the memory limitations of the target embedded sensor platform. This validation should, however, be extended to higher dimensional excerpts to fully evaluate the performance and advantage of multilead delineation over its single-lead counterpart.

Additionally, Table III reports the memory usage and execution time on the Shimmer of the delineation algorithms validated in Table II. In particular, it shows that RMS-based multilead delineation requires up to two times the memory usage of its single-lead counterpart. This is because it must filter the two leads separately for baseline removal, combine them, and finally delineate the resulting signal. Interestingly, both RMSbased implementations exceed the $10 \mathrm{kB}$ RAM memory of the platform. The corresponding results in Tables III and VI have in fact been obtained using an alternative version of the MSP430 microcontroller (MSP430F5438), which is equipped with $16 \mathrm{kB}$ of RAM memory. Moreover, the execution time of the RMSbased methods is considerably higher than that of single-lead delineation, with most of the computational burden dedicated to baseline wander removal (i.e., $26.44 \%$ for cubic spline baseline estimation versus $12.39 \%$ for morphological filtering).

\section{Energy Versus Performance Trade-off Study}

It is widely accepted that wireless communication is the main source of energy consumption in a wireless sensor platform [32]-[34]. Therefore, it is largely preconized to preprocess the sensor data onboard the embedded platform, in order to reduce the amount of information to be wirelessly transmitted to the WBSN sink. This section revisits these assumptions, through analyzing the impact of on-board data processing on the energy consumption of the sensor node and identifying the main contributors to this consumption.

\section{A. Experimental Framework}

This work is based on the Shimmer platform, as a representative state-of-the-art wearable platform, equipped with an ultralow-power microcontroller (Texas Instruments MSP430) and a low-power low-rate IEEE 802.15.4-compliant radio (CC2420). Regarding the wireless communication, our target node implements a simple TCP/IP stack for the CC2420 radio instead of the IEEE 802.15.4 [35]. Thus, in order to fully exploit the low-power capabilities of this radio chip, we implemented a reduced ver-

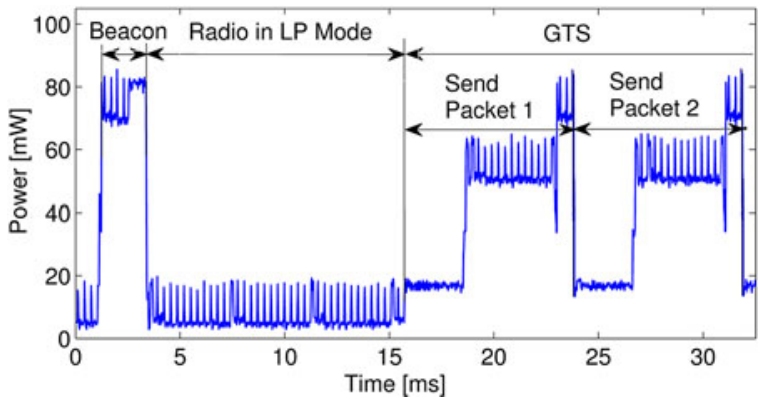

Fig. 2. Power dissipated in Shimmer during sampling, processing and transmission.

sion of the beacon-enabled mode of the IEEE 802.15.4 protocol using guaranteed time slots (GTS) [35] over a star network (i.e., each sensor node can only send data to a designated WBSN coordinator/sink). In this mode, the coordinator broadcasts periodic beacons which are used by the rest of the nodes to synchronize their transmissions with their exclusively allocated GTS. In all the following, the protocol was configured for eight active nodes and GTS long enough to send four full data packets, adjusting the beacon interval to fulfill the bandwidth requirements of the most demanding node. This node is an ECG-sensing device, which samples the ECG signal, processes it according to one of the considered delineation methods, and sends the delineation results to the WBSN coordinator.

To measure the power consumption during operation, we place a $10.3 \Omega$ resistor in the power path of the node. The voltage is then measured using an oscilloscope, and the corresponding current and power consumption are calculated. Fig. 2 depicts the power consumption trace related to a sensing node running a simple streaming application, in which the raw ECG signal is forwarded to the WBSN coordinator without performing any on-board processing. Regarding the radio, three main phases can be distinguished in Fig. 2: beacon reception, low power (LP) mode, and transmission. During beacon reception, the radio first switches to reception mode before the beacon is expected, then receives it. Therefore, the beacon reception phase in Fig. 2 has two clearly distinct parts: 1) the first part lasts for $1.39 \mathrm{~ms}$, has a power consumption of $72.39 \mathrm{~mW}$, and corresponds to a radio in reception mode waiting for the beacon while the microcontroller is idle; 2) the second part lasts for 0.97 $\mathrm{ms}$, has a power consumption of $82.59 \mathrm{~mW}$, and corresponds to a radio in reception mode and a microcontroller awake reading from the radio and analyzing the received beacon. After the beacon reception, the node radio enters an LP mode until the start of its assigned GTS. Finally, during its GTS, the node transmits the ECG signal to the coordinator. Fig. 2 depicts the transmission of two data packets. In every packet transmission, two different zones can be clearly distinguished: 1) the first zone corresponds to the microcontroller sending the full packet to the CC2420 radio chip (which is in idle mode), and consumes $16.8 \mathrm{~mW}$ during $2.85 \mathrm{~ms} ; 2$ ) the second zone corresponds to the radio actually transmitting the packet while the microcontroller switches back to idle mode, and dissipates $51.92 \mathrm{~mW}$ during $4.51 \mathrm{~ms}$. After each packet transmission, the radio is seen to automatically go 
TABLE IV

Average Power Characterization (IN $m W$ ) OF SHIMMER

\begin{tabular}{l|l|l|l}
\hline \hline & CPU & Radio & Total \\
\hline Radio reception $(\mu \mathrm{C}$ idle) & 6.60 & 65.79 & 72.39 \\
\hline Radio reception $(\mu \mathrm{C}$ active $)$ & 16.80 & 65.79 & 82.59 \\
\hline Packet transmission (from $\mu \mathrm{C}$ to radio) & 16.80 & 0.00 & 16.80 \\
\hline Packet transmission (actual radio transmission) & 6.60 & 45.32 & 51.92 \\
\hline Sampling $(\mu \mathrm{C}$ and radio idle) & 6.60 & 0.00 & 6.60 \\
\hline Sampling $(\mu \mathrm{C}$ active and radio idle) & 16.80 & 0.00 & 16.80 \\
\hline
\end{tabular}

TABLE V

AVERAge Timing CHARACTERIZATION (IN $m s$ ) OF SHIMMER

\begin{tabular}{l|c}
\hline \hline Beacon reception (radio in reception and $\mu \mathrm{C}$ idle) & 1.39 \\
\hline Beacon reception (radio in reception and $\mu \mathrm{C}$ active) & 0.97 \\
\hline Packet transmission (from $\mu \mathrm{C}$ to radio) & 2.85 \\
\hline Packet transmission (actual radio transmission) & 4.51 \\
\hline ACK reception & 0.75 \\
\hline
\end{tabular}

TABLE VI

NODE LIFETIME FOR THE DIFFERENT ALGORITHMS

\begin{tabular}{l|l|l|l|l}
\hline \hline & $\begin{array}{l}\text { ECG } \\
\text { Streaming }\end{array}$ & $\begin{array}{l}\text { Single } \\
\text { lead }\end{array}$ & $\begin{array}{l}\text { 2-lead } \\
\text { Morph. } \\
\text { filtering }\end{array}$ & $\begin{array}{l}\text { 2-lead } \\
\text { spline } \\
\text { filtering }\end{array}$ \\
\hline Code execution time $(\%)$ & 0 & 6.78 & 19.17 & 33.22 \\
\hline Packet ready every... $(m s)$ & 304 & 2250 & 2250 & 2250 \\
\hline Beacon interval $(m s)$ & 983 & 7864 & 7864 & 7864 \\
\hline Energy consumption $(m J)$ & 7.70 & 7.42 & 8.68 & 10.12 \\
\hline Lifetime $(h)$ & 134.6 & 139.6 & 119.3 & 102.4 \\
\hline
\end{tabular}

to reception mode, while it waits for an ACK from the WBSN coordinator. The ACK reception lasts for $0.75 \mathrm{~ms}$ and has an associated power consumption of $72.39 \mathrm{~mW}$. During the entire process, the microcontroller is continuously updating the operating system tick counter (every $0.32 \mathrm{~ms}$ ) and the sampling task is reading one sample from the ADC every $4 \mathrm{~ms}$. This process is especially noticeable during the low-power phase of the radio. Table IV summarizes the power consumption of the microcontroller and the radio for the different operating modes of our platform, while Table $\mathrm{V}$ reports the timing information related with the aforementioned phases of the radio.

\section{B. Experimental Results}

Based on the above power characterization, Table VI proposes a comparison of the energy consumption of a Shimmer node running a simple ECG streaming application or the various ECG delineation algorithms considered in this paper. The first row reports the code execution time in percentage of ECG signal acquisition time, as in Table III, while the second row indicates the time interval between the generation of two consecutive data packets of 114 bytes. Given that a sample is represented using 1.5 bytes and that the considered sampling frequency is 250 $\mathrm{Hz}$, the streaming application for instance generates one packet every $304 \mathrm{~ms}$. For the delineation algorithms, on the other hand, 30 bytes of data (i.e., amplitude and timing information of the nine fiducial points of the ECG beat) are generated every time a heart beat is detected. Thus, a packet can store up to three heart beats. Assuming a typical heart beat rate of 80 beats per minute, a full packet is queued for transmission every 2250 $\mathrm{ms}$. The third row shows the beacon interval, which is adjusted according to the time interval between consecutive packets. The fourth row reports the total energy consumption per second for every algorithm. Finally, the last row is directly calculated from the fourth one, assuming the energy supply of the used platform is a $280 \mathrm{mAh}$ Li-ion battery@3.7V.

Table VI shows that the total energy consumption increases with the computational burden of the delineation algorithm. Surprisingly, it also evidences that even the computationally light single-lead delineation algorithm, which only consumes $6.78 \%$ of the CPU time and significantly reduces the amount of data to transmit (from 3.29 to 0.44 packets per second), offers a marginal lifetime extension with respect to the plain ECG streaming. These results highlight that, for WBSN applications, the radio is not always responsible for most of the energy consumption of the node, as widely assumed. To understand the limited lifetime extension provided by embedded signal preprocessing, we subsequently characterize and analyze the energy consumption breakdown of the considered platform. The two main sources of energy consumption are:

1) The Radio: The energy consumption of the radio depends on the medium access control (MAC) protocol [35], which adds communication overhead to control the access to the shared medium. The fraction of energy consumption due to the used MAC protocol only represents $1.3 \%$ for the cubic spline delineation, and $14.3 \%$ for streaming. Therefore, the considered GTS-based protocol is a good choice for the continuous ECG monitoring with periodic reporting scenario. It is worthwhile mentioning that this energy characterization was done for lineof-sight communications between the sensing node and the WBSN coordinator. A detailed study of the impact of various body-area propagation conditions should be performed. It is, however, outside of the scope of this paper.

2) The Microcontroller: The consumption due to the CPU has a significant impact on the node lifetime for delineation algorithms that require more computational power than wireless ECG streaming. Except for single-lead delineation, the increased CPU activity leads to a shorter node lifetime, although the radio energy consumption is decreased by a factor of 8.6 (from streaming to delineation). Thus, the code execution can be very expensive if the power consumption of the microcontroller is high or the algorithm to be executed has not been carefully optimized for the target platform. Therefore, a careful study must be done when choosing or designing the microcontroller of each final WBSN platform. Indeed, it is key to take into account the power consumption in active mode as well as the possibility of having a low voltage operation or using low-power modes that disable some parts of the microcontroller when they are not used.

Nevertheless, for the Shimmer platform, the dominant process of the microcontroller in terms of energy consumption is not the code execution. As illustrated in Fig. 3, most of the energy consumption is due to sampling the ECG signal (i.e., $65.2 \%$ for multilead delineation with cubic spline baseline estimation and $85.7 \%$ for the streaming scenario). Therefore, the sampling process can be very expensive in terms of energy consumption depending on the hardware implementation and the sampling frequency. In this particular case, for the considered sampling frequency of $250 \mathrm{~Hz}$, it was not possible to turn the internal 


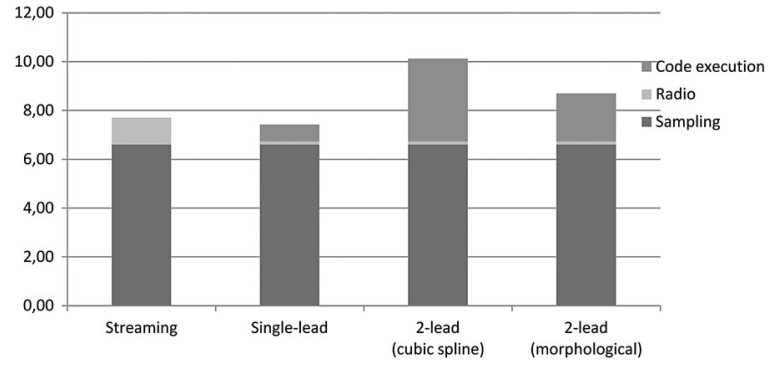

Fig. 3. Breakdown of energy consumption of Shimmer.

reference voltage of the A/D converters off and on between two consecutive samples, because the time that the internal voltage regulator takes to reach the appropriate level for the conversion is longer than the sample duration. Therefore, the voltage reference is always active, increasing significantly the total energy consumption. Hence, a careful choice of an ultra-low-power $\mathrm{A} / \mathrm{D}$ converter can potentially decrease to a large extent the energy consumption of the whole system, as the sampling process has proven to be the greatest energy-draining task in the design of our wearable ECG WBSN system.

\section{CONCLUSION}

This work has evidenced the feasibility of real-time multilead ECG delineation on a state-of-the-art commercial wearable WBSN platform, namely Shimmer. Furthermore, it has shown that the considered RMS-based multilead delineation performs equivalently to the best single-lead delineation for the 2-lead QTDB database, within a fraction of a sample duration of the CSE tolerances. This confirmed that this multilead approach alleviates the dependency of delineation results on specific lead or lead systems, and on expert intervention. Moreover, the two evaluated baseline wander removal approaches, namely the cubic spline baseline estimation and the morphological filtering, were shown to provide different trade-offs between positive predictivity, on the one hand, and computational burden and memory footprint, on the other hand. This work also proposed a comprehensive evaluation of the Shimmer's energy consumption for a simple raw ECG streaming application and the various delineation implementations investigated. The resulting energy consumption breakdown showed: 1) provided an optimized schedule-based MAC protocol and corresponding optimized radio shutdown, a single-lead embedded delineation only offers a marginal node lifetime extension; 2) this marginal advantage vanishes for multilead approaches, which entail up to five times increase of CPU usage; 3 ) the ECG sampling is the greatest energy-draining task for the considered sampling frequency. Accordingly, our system-level exploration suggests that node lifetime extension can be achieved provided: 1) an ultralow-power A/D converter and associated architecture is used to rationalize the ECG sampling cost; 2) a truly ultra-low-power microcontroller is chosen to carry out signal preprocessing; 3 ) last, but not least, a sensible algorithmic and software optimization of the embedded signal processing is performed to take into account the limited processing and storage resources of wireless sensor nodes.

\section{REFERENCES}

[1] World Health Organization. (2009). Cardiovascular diseases, [Online]. Available: http://www.who.int/topics/cardiovascular diseases/

[2] L. Sörnmo and P. Laguna, Bioelectrical Signal Processing in Cardiac and Neurological Applications. Amsterdam, The Netherlands: Elsevier Academic Press, 2005, ch. 7.

[3] U. R. Acharya, J. S. Suri, J. A. E. Spaan, and S. M. Krishnan, Advances in Cardiac Signal Processing. New York: Springer-Verlag, 2010, ch. 2-4.

[4] P. Laguna, R. Jané, and P. Caminal, "Automatic detection of wave boundaries in multilead ECG signals: Validation with the CSE database," Comput. Biomed. Res., vol. 27, no. 1, pp. 45-60, Feb. 1994.

[5] C. Li, C. Zheng, and C. Tai, "Detection of ECG characteristic points using wavelet transforms," IEEE Trans. Biomed. Eng., vol. 42, no. 1, pp. 21-28, Jan. 1995.

[6] J. S. Sahambi, S. Tandon, and R. K. P. Bhatt, "Using wavelet transform for ECG characterization," IEEE Eng. Med. Biol., vol. 16, no. 1, pp. 77-83, 1997.

[7] J. P. Martinez et al., "A wavelet-based ECG delineator: evaluation on standard databases," IEEE Trans. Biomed. Eng., vol. 51, no. 4, pp. 570581, Apr. 2004.

[8] H. Vullings, M. Verhaegen, and H. Verbruggen, "Automated ECG segmentation with dynamic time warping," in Proc. IEEE EMBC, 1998, pp. 163-166.

[9] Z. Dokur, T. Olmez, E. Yazgan, and O. Ersoy, "Detection of ECG waveforms by neural networks," Med. Eng. Phys., vol. 19, no. 8, pp. 738-741, 1997.

[10] S. Graja and J.-M. Boucher, "Hidden Markov tree model applied to ECG delineation," IEEE Trans. Instrum. Meas., vol. 54, no. 6, pp. 2163-2168, 2005.

[11] Y. Sun, K. L. Chan, and S. M. Krishan, "Characteristic wave detection in ECG signal using morphological transform," BMC Cardiovasc. Disorders, vol. 5 , no. 28, 2005.

[12] Toumaz Technology. (2009). [Online]. Available: http://www.toumaz. com/public/news.php?id=92

[13] Corventis, 2009. [Online]. Available: http://www.corventis. com/AP/nuvant.asp

[14] R. F. Yazicioglu, T. Torfs, J. Penders, I. Romero, H. Kim, P. Merken, B. Gyselinckx, H. J. Hoo, and C. V. Hoof, "Ultra-low-power wearable biopotential sensor nodes," in Proc. IEEE EMBC, Sep. 2009.

[15] Shimmer Sensor Platform, [Online]. Available: http://shimmerresearch.com.

[16] N. Boichat, N. Khaled, F. Rincón, and D. Atienza, "Wavelet-based ECG delineation on a wearable embedded sensor platform," in Proc. Conf. Body Sens. Netw., 2009, pp. 256-261.

[17] Z. Ihara et al., "Atrial repolarization as observable during the PQ interval," J. Electrocardiol., vol. 39, no. 3, pp. 290-297, 2006.

[18] M. Llamedo Soria et al., "A multi-lead wavelet-based ECG delineator based on the RMS signal," in Proc. Comput. Cardiol., 2006, pp. 153-156.

[19] F. Rincón, N. Boichat, V. Barbero, N. Khaled, and D. Atienza, "Multi-lead wavelet-based ECG delineation on a wearable embedded sensor platform," in Proc. Comput. Cardiol., 2009, vol. 36, no. 9, pp. 289-292.

[20] R. Almeida, J. P. Martínez, A. P. Rocha, and P. Laguna, "Multi-lead ECG delineation using spatially projected leads from wavelet transform loops," IEEE Trans. Biomed. Eng., vol. 56, no. 8, pp. 1996-2005, Aug. 2009.

[21] A. Illanes-Manriquez, "An automatic multi-lead electrocardiogram segmentation algorithm based on abrupt change detection," in Proc. IEEE EMBC, 2010, pp. 2334-2337.

[22] C. R. Meyer and H. N. Keiser, "Electrocardiogram baseline estimation and removal using cubic splines and space-state computation techniques," Comp. Biol. Res., vol. 10, pp. 459-470, 1977.

[23] Y. Sun et al., "ECG signal conditioning by morphological filtering," Comp. Biol. Med., vol. 32, no. 6, pp. 465-479, 2002.

[24] P. Laguna et al., "A database for evaluation of algorithms for measurement of QT and other waveform intervals in the ECG," in Proc. Comput. Cardiol., Sep. 1997, pp. 673-676, [Online]. Available: http://www.physionet.org/physiobank/database/qtdb/doc/

[25] Testing and reporting performance results of cardiac rhythm and ST-segment measurement algorithms, ANSI/AAMI/ISO EC57:1998/(R)2008.

[26] MSP430 microcontroller, [Online]. Available: http://www.ti.com/msp430 
[27] D. Culler, "Tinyos: Operating system design for wireless sensor networks," Sensors, pp. 41-49, 2003.

[28] FreeRTOS real-time operating system, [Online]. Available: http://www.freertos.org/ 2011.

[29] Code Composer Essentials, [Online]. Available: http://www.ti.com/cce

[30] T. J. Rolfe, "On a fast integer square root algorithm," SIGNUM Newsl., vol. 22 , no. 4,1987

[31] C.S.E.W. Party, "Recommendations for measurement standards in quantitative electrocardiography," Eur. Heart J., vol. 6, pp. 815-825, 1985.

[32] G. Mathur, P. Desnoyers, D. Ganesan, and P. Shenoy, "Ultra-low power data storage for sensor networks," in Proc. Int. Conf. Inf. Process. Sens. Netw., 2006, pp. 374-381.

[33] K. Sohrabi, J. Gao, V. Ailawadhi, and G. Pottie, "Protocols for selforganization of a wireless sensor network," IEEE Pers. Commun., vol. 7, no. 5, pp. 16-27, Oct. 2000.

[34] M. I. Brownfield, A. S. Fayez, T. M. Nelson, and N. Davis, "Crosslayer wireless sensor network radio power management," in Proc. IEEE Wireless Commun. Netw. Conf., Apr. 2006, vol. 2, pp. 1160-1165.

[35] IEEE 802.15.4 Std. Wireless Medium Access Control (MAC) and Physical Layer (PHY) specifications for low-rate wireless personal area networks (LR-WPANs), IEEE, 2003.

[36] Fluke, MPS450 ECG/Arrhythmia Simulator, [Online]. Available: http://www.flukebiomedical.com/Biomedical/usen/BiomedicalTest/Simulators-\&-Controllers/MPS450.htm?PID=56424

[37] L. Schamroth, The Disorders of Cardiac Rhythm. Oxford, U.K.: Blackwell Scientific Publications, 1971.

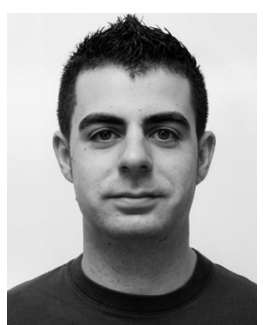

Francisco Rincón received the M.Sc. degree in computer science and engineering from the Complutense University of Madrid (UCM), Spain, in 2006.

$\mathrm{He}$ is currently a Teaching Assistant of computer science with the Department of Computer Architecture and Automation, UCM. His current research interests include energy-aware optimizations for WBSN. In this field, he is co-author and hold U.S. patent.

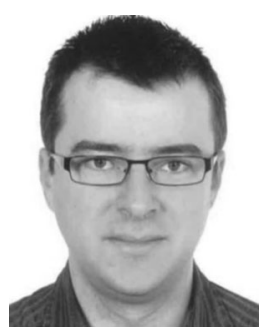

Joaquin Recas received the M.Sc. degree in computer science and engineering from the Universidad Autónoma de Madrid, Spain, in 2000 and the M.Sc. degree in electronics and the Ph.D. degree in computer science from the Complutense University of Madrid (UCM), Spain, in 2004 and 2006, respectively.

$\mathrm{He}$ is currently an Assistant Professor with the Department of Computer Architecture and Automation, UCM. His current research interests include low power embedded systems applied to real-time signal

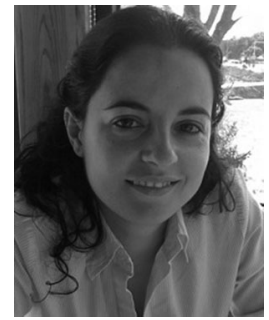

Nadia Khaled received the M.Sc. degree in electrical engineering from ENSEEIHT, Toulouse, France, in 2000, and the Ph.D. degree in electrical engineering from the Katholieke Universiteit Leuven, Belgium, in 2005 .

From 2000 to 2005, she was with the wireless research group of the Interuniversity Microelectronics Center (IMEC), Leuven. From 2005 to 2006, she was a Postdoctoral Researcher at ETH Zurich, Switzerland. Since 2006, she is with Ecole Polytechnique Féderale de Lausanne (EPFL), Switzerland, and has held the position of Visiting Assistant Professor at the University of Carlos III Madrid (UC3M) from 2006 to 2009. From 2010, she is Senior Scientist at EPFL, where she is leading a joint NestlPFL project on non-intrusive, intelligent and wearable sensors to help monitoring the health, well-being and nutrition of the elderly.

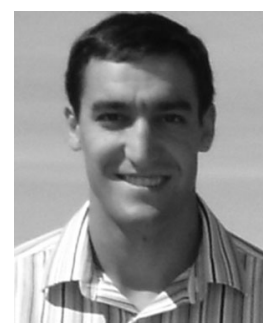

David Atienza received the M.Sc. and Ph.D. degrees in computer science and engineering from Complutense University of Madrid (UCM), Spain, and Inter-University Microelectronics Center (IMEC), Belgium, in 2001 and 2005, respectively.

$\mathrm{He}$ is currently a Professor of EE and Director of the Embedded Systems Laboratory (ESL) at Ecole Polytechnique Fédérale de Lausanne (EPFL), Switzerland, and Adjunct Professor at the Computer Architecture Department of UCM. His research interests focus on system-level design methodologies for high-performance MPSoC and embedded systems, including new system architectures for wireless body sensor networks, 2D/3D thermal-aware design for MPSoCs, HW/SW reconfigurable processing systems, dynamic memory optimizations, and NoC design. In these fields, he is coauthor of more than 160 publications in peer-reviewed international journals and conferences, and several book chapters. He holds two U.S. patents.

Dr. Atienza has received a Best Paper Award at the VLSI-SoC 2009 conference, and three Best Paper Award Nominations at the WEHA-HPCS 2010 , ICCAD 2006, and DAC 2004 conferences. He is Associate Editor of IEEE TRANSACTIONS ON COMPUTER-AIDED DESIGN OF CIRCUITS AND SYSTEMS and Elsevier Integration. He has been a member of the Executive Committee of the IEEE Council on EDA since 2008, and member of the Board of Governors of the IEEE Circuits and Systems Society (CASS), since 2010. 